\title{
Hubbard Gap Modulation in Vanadium Dioxide Nanoscale Tunnel Junctions
}

\author{
Magdalena Huefner, ${ }^{, \dagger}$ Ram Krishna Ghosh, ${ }^{\ddagger}$ Eugene Freeman, ${ }^{\ddagger}$ Nikhil Shukla, ${ }^{\ddagger}$ Hanjong Paik, ${ }^{\S}$ \\ Darrell G. Schlom, ${ }^{\S}, \|$ and Suman Datta ${ }^{*}$ \\ ${ }^{\dagger}$ Department of Physics, Harvard University, Cambridge, Massachusetts 02138, United States \\ ${ }^{\ddagger}$ Department of Electrical Engineering, The Pennsylvania State University, University Park, Pennsylvania 16802, United States \\ ${ }^{\S}$ Department of Materials Science and Engineering, Cornell University, Ithaca, New York 14853, United States \\ "Kavli Institute at Cornell for Nanoscale Science, Ithaca, New York 14853, United States
}

ABSTRACT: We locally investigate the electronic transport through individual tunnel junctions containing a $10 \mathrm{~nm}$ thin film of vanadium dioxide $\left(\mathrm{VO}_{2}\right)$ across its thermally induced phase transition. The insulator-to-metal phase transition in the $\mathrm{VO}_{2}$ film collapses the Hubbard gap (experimentally determined to be 0.4 $\pm 0.07 \mathrm{~V}$ ), leading to several orders of magnitude change in tunnel conductance. We quantitatively evaluate underlying transport mechanisms via theoretical quantum mechanical transport calculations which show excellent agreement with the experimental results.

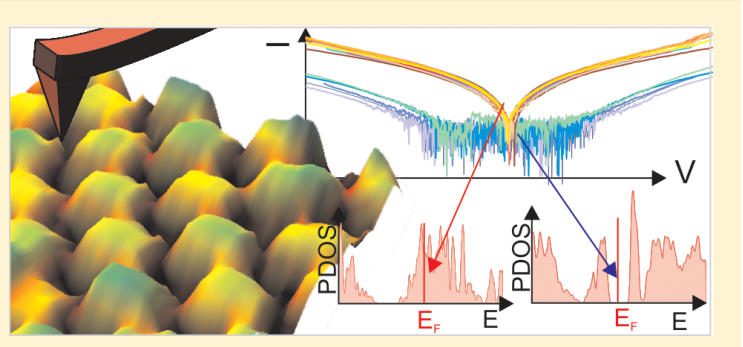

KEYWORDS: Atomic force microscopy, metal-insulator transition, vanadium dioxide, density functional theory, non-equilibrium Green's function, tunnel junction

$\mathrm{V}$ anadium dioxide $\left(\mathrm{VO}_{2}\right)$ is a correlated electron material that exhibits a metal-to-insulator transition (MIT) with up to 6 orders of magnitude change in in-plane resistivity near room temperature $(\sim 340 \mathrm{~K})$ in unstrained films. ${ }^{1}$ The MIT can be externally triggered using thermal, ${ }^{2}$ electronic, ${ }^{3}$ optical, ${ }^{4}$ or strain stimuli. ${ }^{5}$ It has also been demonstrated by time-resolved optical pumping that this transition is as fast as $75 \mathrm{fs}^{4,6}$ In the insulating state, $\mathrm{VO}_{2}$ has an optically measurable Hubbard gap of $0.6 \mathrm{eV},{ }^{7}$ which abruptly collapses as $\mathrm{VO}_{2}$ undergoes an insulator-to-metal transition. The collapse of the Hubbard gap and ultrafast switching holds promise for high speed correlated tunnel transistors. ${ }^{8}$ This increases the interest to investigate nanostructures that contain $\mathrm{VO}_{2}$.

In this work we investigate nanoscale tunnel junctions that contain a thin layer of $\mathrm{VO}_{2}$. Our structures incorporate an electrically insulating film on top of a thin $\mathrm{VO}_{2}$ film between metallic contacts. This layout allows us to directly study the out-of-plane electronic transport behavior through a thin film of $\mathrm{VO}_{2}$ throughout its phase transition.

When $\mathrm{VO}_{2}$ is in the metallic state, such a metal-insulatormetallic $\mathrm{VO}_{2}$-metal structure essentially acts as a metalinsulator-metal tunnel junction. In this state direct tunneling is expected to be the dominating underlying transport mechanism $^{9}$ as electrons can tunnel directly from one metallic layer, through the insulating barrier into the next metallic layer (Figure 1a). When $\mathrm{VO}_{2}$ is in its insulating state, a bandgap $E_{\mathrm{g}}$ opens in the $\mathrm{VO}_{2}$ film (Figure 1b). This bandgap eliminates states near the Fermi level previously used for the electronic transport, thus significantly lowering the tunneling current

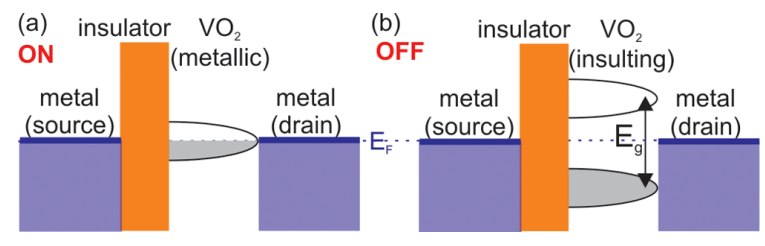

Figure 1. Schematic bandstructure for a metal-insulator $-\mathrm{VO}_{2}$ layered structure when (a) $\mathrm{VO}_{2}$ is in an electrically metallic state and (b) in an insulating state. $E_{\mathrm{F}}$ is the Fermi energy. Solid regions indicate filled electronic states.

through the structure. This bandstructure is fundamentally different from conventional semiconductor based tunnel junctions where the electronic transport is dominated by band-bending and the Fermi level movement. ${ }^{9}$

Such metal-tunnel barrier- $\mathrm{VO}_{2}$ tunnel junctions were first demonstrated by Martens et al., ${ }^{10}$ on devices with diameters of several hundred micrometers and using atomic layer deposition (ALD) at temperatures of $200{ }^{\circ} \mathrm{C}$ and higher. Martens et al. could show approximately 1 order of magnitude conductance change when thermally driving these devices across the MIT.

In this work, we investigate the electronic transport through nanoscale tunnel junctions fabricated via low temperature $\left(110{ }^{\circ} \mathrm{C}\right)$ ALD. We locally investigate the temperature

Received: June 3, 2014

Revised: August 25, 2014 
dependence of the electrical conductance for individual nanopillars and observe a change in conductance of roughly 2 orders of magnitude as the $\mathrm{VO}_{2}$ film goes through the MIT. To quantitatively understand the quantum transport in these tunnel junction devices, we carry out nonequilibrium transport calculations under the framework of density functional theory (DFT) combining with nonequilibrium Green's function (NEGF) methods.

The sample under investigation is a multilayer nanopillar array of $\mathrm{VO}_{2}$ tunnel junctions. A schematic side view is shown in Figure 2a. The substrate consists of electrically conducting

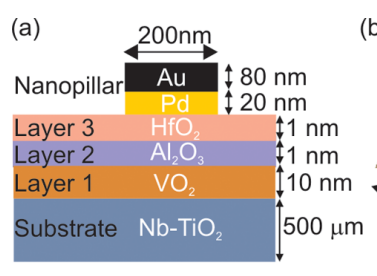

(c)

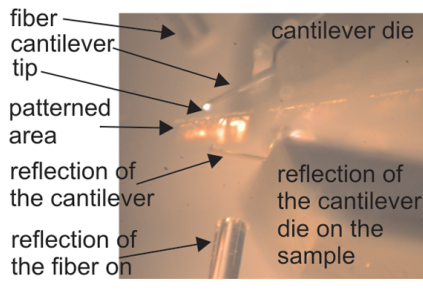

Figure 2. (a) Schematic side view of the sample showing the multilayer structure of the thin film and the nanopillar (not to scale). (b) In situ topography scan of the area under investigation recorded via atomic force microscopy. The single pillars are clearly discernible. (c) Overview photograph of the measurement setup. (d) Schematic of the measurement setup (not to scale).

$500 \mu \mathrm{m}$ thick (001) Nb-doped $\mathrm{TiO}_{2}$ from CrysTec. Onto this substrate we deposit a $10 \mathrm{~nm} \pm 5 \AA$ thick epitaxial $\mathrm{VO}_{2}$ film via reactive oxide molecular beam epitaxy using a Veeco GEN10 system (layer 1$).{ }^{8}$ Previous studies ${ }^{11}$ have shown that annealing $\mathrm{VO}_{2}$ at temperatures above $150{ }^{\circ} \mathrm{C}$ can cause irreversible metallic regions to form on the $\mathrm{VO}_{2}$ surface, therefore prompting us to select low-temperature ALD as a means to preserve the $\mathrm{VO}_{2}$ tunneling interface quality. The layers 2 and 3 -together called the dielectric-are deposited in a Cambridge Savannah 200 ALD system. Layer 2 consists of $1 \mathrm{~nm} \pm$ $1 \AA$ thick $\mathrm{Al}_{2} \mathrm{O}_{3}$ deposited at a substrate temperature of $100{ }^{\circ} \mathrm{C}$ using the metal organic precursor trimethylaluminum. Layer 3 consists of $1 \mathrm{~nm} \pm 1 \AA$ thick $\mathrm{HfO}_{2}$ deposited at a substrate temperature of $110{ }^{\circ} \mathrm{C}$ using tetrakis(dimethylamino)hafnium metal organic precursors with $\mathrm{H}_{2} \mathrm{O}$ as the oxygen source. We deposit a bilayer photoelectric resist consisting of $150 \mathrm{~nm}$ methyl methacrylate (MMA) and $150 \mathrm{~nm}$ poly(methyl methacrylate) (PMMA) and create a positive resist mask for the nanopillars using electron beam lithography. The resist is developed in 1:3 MIBK-IPA (methyl isobutyl ketoneisopropyl alcohol) followed by rinsing in deionized water. We deposit $20 \mathrm{~nm}$ palladium $(\mathrm{Pd})$ followed by $80 \mathrm{~nm}$ gold $(\mathrm{Au})$ via electron beam evaporation onto this positive resist mask. The $\mathrm{Pd} / \mathrm{Au}$ metal stack contacts the dielectric only in isolated regions exposed by the electron beam lithography mask. A standard lift-off procedure (solvent stripper "RemoverPG" at $70{ }^{\circ} \mathrm{C}$ for $10 \mathrm{~min}$ ) is used to remove the resist mask, leaving only the isolated $\mathrm{Pd} / \mathrm{Au}$ nano pillars on top of Layer 3. Each nanopillar has a height of $100 \mathrm{~nm}$ and a diameter of $200 \mathrm{~nm}$.

Figure $2 \mathrm{~b}$ shows an in situ scan of the topography of the sample, providing a top view onto an array of nanopillars. The individual, round nanopillars in a regular array are clearly discernible. The pillars have an average diameter of about $200 \mathrm{~nm}$.

We record electrical and topographic measurements using a custom built low-temperature scanning probe microscope. The cantilever used is a $\mathrm{Cr}-\mathrm{Au}$ MikroMasch NSC-16 with a nominal tip radius of $35 \mathrm{~nm}$. The deflection of the cantilever is measured via a laser-based interferometer system using a fiber that is placed directly above the cantilever as visible in Figure $2 \mathrm{c}$. The cantilever tip is positioned above the region on the sample that is patterned with nanopillars. We align the tip to be centered with respect to this patterned region by using an optical microscope and the reflection of the cantilever on the sample under favorable light conditions (Figure 2c). After the alignment is accomplished, the system is evacuated, and all measurements are performed without breaking the vacuum and at a controlled temperature.

We electrically contact the substrate of the sample which acts as a back contact electrode. The top contact to an individual nanopillar is established by making mechanical contact between the cantilever tip and the nanopillar under investigation (Figure 2d). To do so we press the cantilever tip onto the nanopillar with a constant cantilever deflection. We then apply a voltage to the electrically conducting cantilever and measure the current that flows from the tip through the nanopillar to the back electrode.

An advantage of using the cantilever tip of a fully functional atomic force microscope (AFM) to electrically contact a nanopillar is that it also allows us to image the topography after each electrical measurement. This allows us to perform measurements on several distinct nanopillars that we can identify and track over the complete temperature range of $80 \mathrm{~K}$ without breaking the vacuum. We can also ensure the topographic integrity of the nanopillar under investigation and image undesired particles (if any) that might be present on the sample surface. A typical topography scan is shown in Figure $2 b$.

Using this method we record the current $I$ through a nanopillar versus sweeps of the applied voltage $V$, so-called $I V$ traces. The raw data show a constant instrument originated current offset of about $20 \mathrm{pA}$ for which we correct in all measurement data. For each temperature and each nanopillar, we record 20 individual $I V$ traces under identical conditions, the average of which is then used for further evaluation.

Figure $3 \mathrm{a}-\mathrm{c}$ shows $I V$ traces recorded over a temperature range from 260 to $360 \mathrm{~K}$ in $5 \mathrm{~K}$ temperature steps for three individual nanopillars (A-C). We observe two distinctly different shapes of $I V$ traces: traces recorded above a transition temperature $T_{\mathrm{c}}$ show a continuous increase of current with applied voltage. This is the signature shape of the $I V$ trace in the ON state where direct tunneling takes place from the Pdlayer through the tunnel barrier posed by the dielectric into the metallic $\mathrm{VO}_{2}$ layer. Typical currents are of the order of $1 \mathrm{nA}$ at $V=0.4 \mathrm{~V}$.

Below $T_{c}$ the shape of the $I V$ traces abruptly changes and shows a region of current blockade around $0 \mathrm{~V}$. The width of this current blockade region (Hubbard gap) is $0.4 \pm 0.07 \mathrm{~V}$. Outside of this blockade region, the current through the structure rises with increasing applied voltage. This is the 

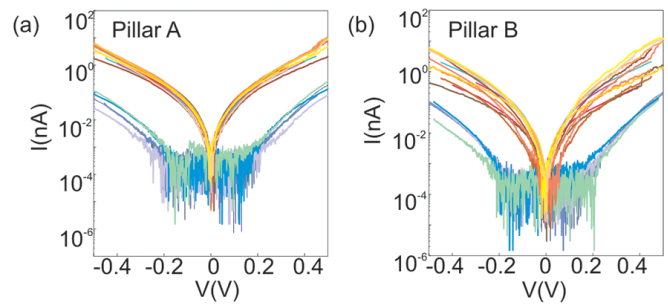

(c)
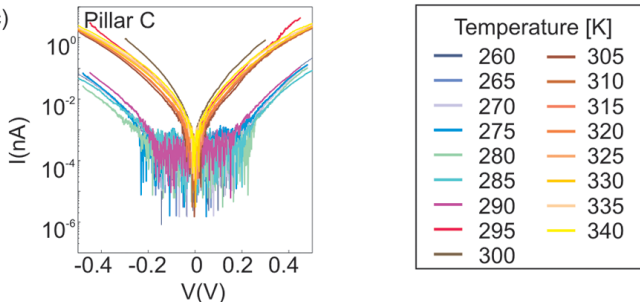

Figure 3. $(\mathrm{a}-\mathrm{c}) I V$ traces for temperatures between 260 to $340 \mathrm{~K}$ in 5 $\mathrm{K}$ steps as indicated in the legend for three different pillars (A, B, C).

signature shape of the $I V$ trace in the OFF state where typical currents are of the order of $0.02 \mathrm{nA}$ at $V=0.4 \mathrm{~V}$.

The $T_{\mathrm{c}}$ for pillars $\mathrm{A}$ and $\mathrm{B}$ is $285 \pm 5 \mathrm{~K}$ and $290 \pm 5 \mathrm{~K}$ for Pillar C. A slight variation in $T_{\mathrm{c}}$ for different nanopillars could be due to a variation in local strain, temperature, or film thickness. We extract the conductance $G\left(V_{\mathrm{E}}\right)$, here defined as $G\left(V_{\mathrm{E}}\right)=\left(I\left(V_{\mathrm{E}}\right)\right) / V_{\mathrm{E}}$, at distinct evaluation voltages $V_{\mathrm{E}}$ (Figure $4 a)$ and plot its value versus the measurement temperature
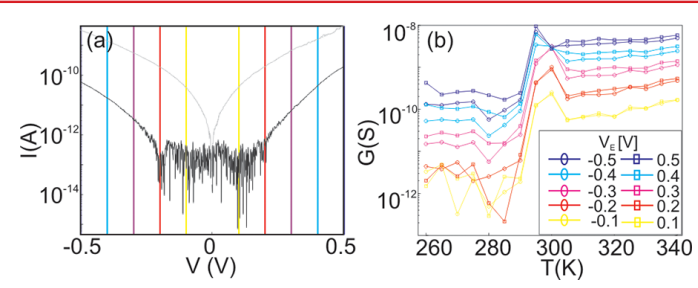

Figure 4. (a) Typical traces for the current vs voltage when the $\mathrm{VO}_{2}$ is in the insulating (black) and conducting state (gray). $G_{\mathrm{E}}=G\left(V_{\mathrm{E}}\right)$ is extracted at the values of $V_{\mathrm{E}}$ as indicated by solid lines for all measured temperatures. (b) Resulting trace of $G_{\mathrm{E}}$ vs $T$ of an individual pillar for $V_{\mathrm{E}}$ in volts as indicated in the legend.

(Figure 4b). For each individual pillar we observe that for both regions, $T>T_{\mathfrak{c}}$ and $T<T_{\mathcal{c}}$ the conductance is nearly independent of temperature. This indicates that the dominant temperature dependent process that influences the conductance is the phase transition in $\mathrm{VO}_{2}$ at $T_{\mathrm{c}}$. All other transport processes show only a very weak temperature dependence. At $T_{c}$ the conductance changes by approximately 2 orders of magnitude. The precise magnitude of the change depends on the voltage $V_{\mathrm{E}}$ at which the comparison is carried out. Figure 4 shows that, when $\mathrm{VO}_{2}$ undergoes a MIT at $T_{\mathcal{c}}$ a large change in tunnel conductance at low applied voltage takes place. This is a promising transport property that could be used to achieve a steep slope switching characteristics in future device applications.

To make a direct comparison between the measurements and the quantum mechanical transport in $\mathrm{VO}_{2}$ based tunnel junction devices, we carry out nonequilibrium transport calculations by using density functional theory combining with the non-equilibrium Green's function formalism, as implemented in the simulator Atomistic Toolkit. ${ }^{12}$ Before performing the transport calculations, first-principle calculations of both high-temperature rutile (Figure 5a) and low- (a)

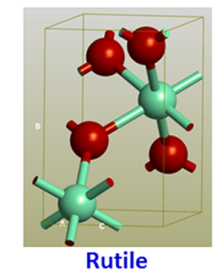

(b)

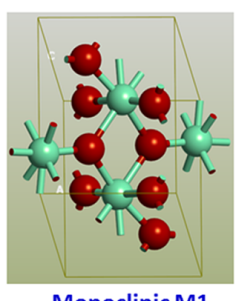

(c)

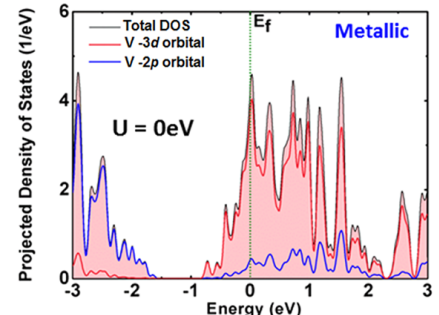

(d)

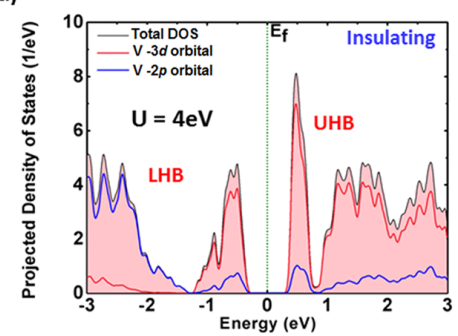

Figure 5. (a) $\mathrm{VO}_{2}$ in its high-temperature rutile crystal structure and (b) its corresponding PDOS which shows a metallic nature. (c) The low-temperature $\mathrm{Ml}$ crystal structure of $\mathrm{VO}_{2}$, which gives a metallic nature in conventional DFT but (d) opens a band gap of $0.56 \mathrm{eV}$ through a DFT $+\mathrm{U}$ calculation. HB is the Hubbard band, and DOS is the density of states.

temperature monoclinic (M1) (Figure 5c) crystal structure (in bulk form) of $\mathrm{VO}_{2}$ are carried out by $\mathrm{DFT}+\mathrm{U}$ where $U$ is the Hubbard electron-electron correlation parameter. In our computations, different lattice parameters of both $\mathrm{VO}_{2}$ crystal structures as determined by Eyert et al. ${ }^{13}$ have been considered. We adopt the Perdew-Burke-Ernzerhof functional under generalized gradient approximation for the exchange correlation along with double- $\zeta$-double polarized basis set for the electron wave function. These electron wave functions are usually comparable to well-converged plane wave basis sets, whereas the core electrons are defined by norm-conserving pseudopotentials. The computed projected density of states (PDOS) for both crystal structures is shown in Figure 5. It is wellunderstood that the high-temperature rutile crystal structure leads to metallic behavior. ${ }^{13,14}$ Lowering of the crystal symmetry from rutile to $\mathrm{M} 1$ results in a formation of $\mathrm{V}-\mathrm{V}$ dimers along the rutile $c$-axis, which splits the nonbonding $d$ orbitals into occupied and unoccupied states. ${ }^{13,14}$ Due to the Mott insulator nature of the $\mathrm{M} 1 \mathrm{VO}_{2}$, the conventional DFT cannot open a band gap. With the addition of Hubbard U term, the $d$-orbitals split into a lower Hubbard band (LHB) and an upper Hubbard band (UHB) and eventually produce a MottHubbard gap. The electronic state as projected in our PDOS computations agrees well with the orbital distributions as shown by Quackenbush et al. ${ }^{14}$ From our first-principles study, we observe that $U=4 \mathrm{eV}$ produces a Mott gap of $0.56 \mathrm{eV}$, which is in excellent agreement with the experimental as well as previous theoretical results of $0.6 \mathrm{~V} .^{7,13,15,16}$ Therefore, we considered DFT $+\mathrm{U}$ instead of conventional DFT along with NEGF formalism in our transport calculations of the M1 phase. 

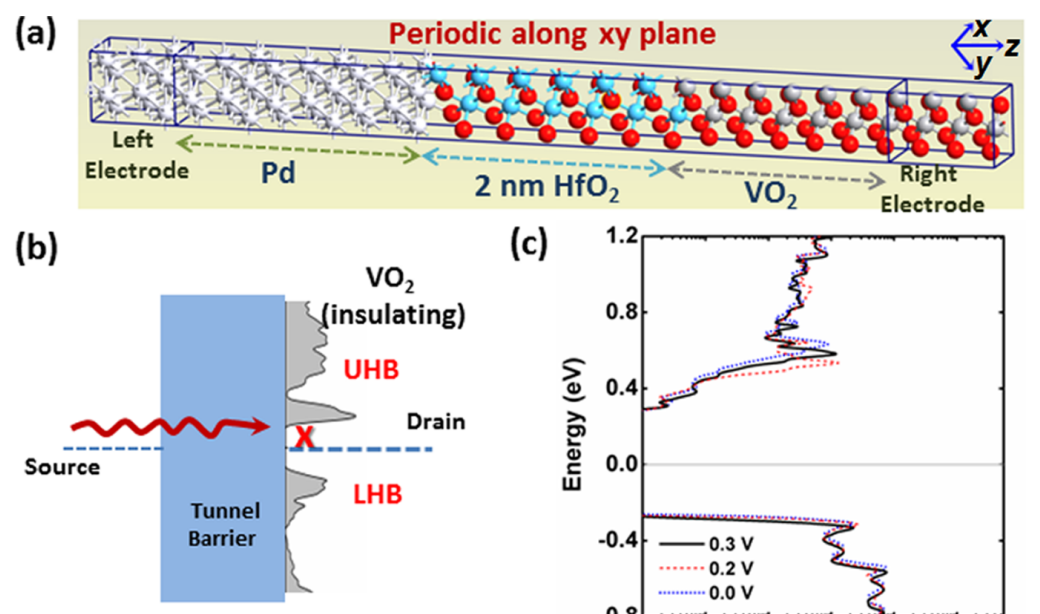

(c)
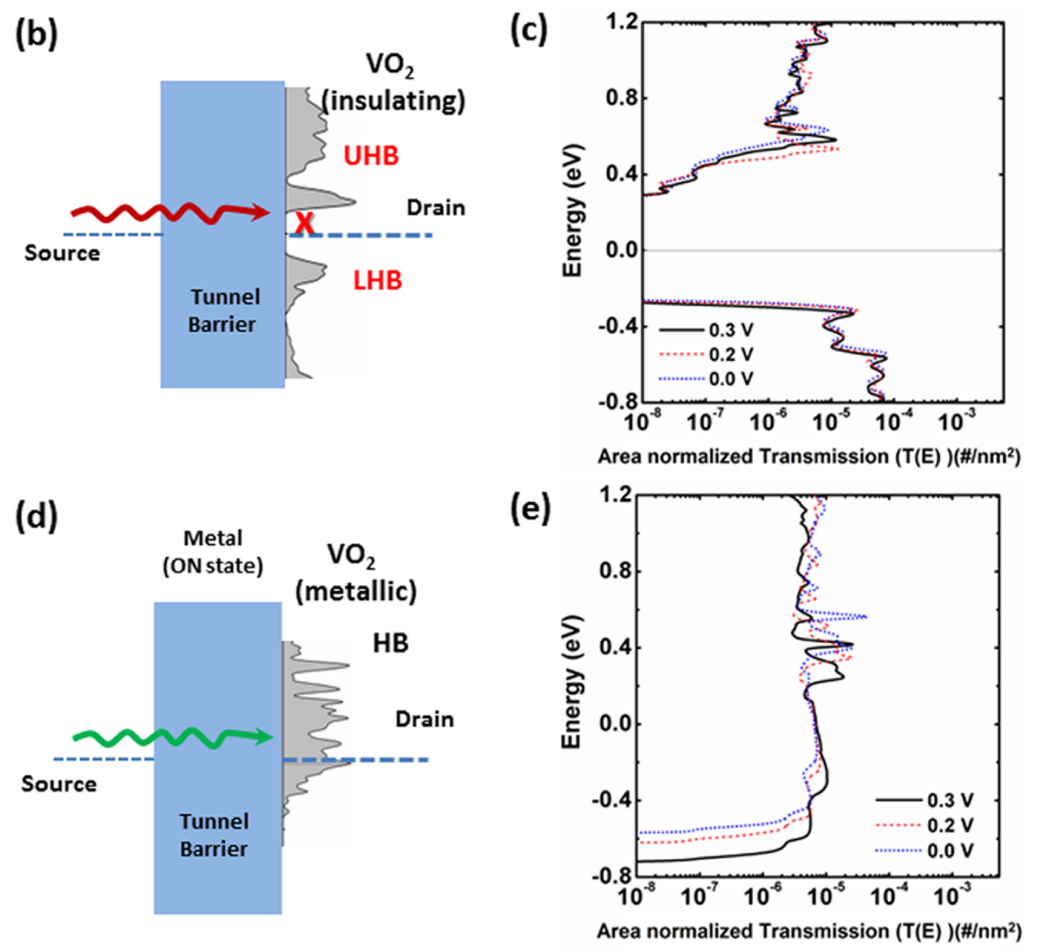

Figure 6. (a) Atomistic structure of $\mathrm{VO}_{2}$ based tunnel junction where the transport direction is along the $z$-axis and the direction perpendicular to the $z$-axis ( $x y$-plane) is periodic in nature. (b) The schematic depicting the tunneling transport mechanism and (c) the corresponding transmission (normalized to the area) when $\mathrm{VO}_{2}$ is in the insulating state. (d, e) Schematic and the corresponding transmission (normalized to the area) when $\mathrm{VO}_{2}$ is in its metallic state.

The atomistic view of the $\mathrm{VO}_{2}$ based tunnel junction device used for our transport calculations is shown in Figure 6a. We consider a simplified device architecture by implementing a 2 $\mathrm{nm}$ thick $\mathrm{HfO}_{2}$ film instead of two different dielectric materials as a tunnel barrier in order to reduce the computational cost by minimizing the number of different interfaces. The $\mathrm{VO}_{2}$ thickness is $2.9 \mathrm{~nm}$ for M1 and $2.8 \mathrm{~nm}$ for the rutile crystal structure where we consider the low-temperature M1 crystal structure for the $\mathrm{VO}_{2}$ 's OFF state below $T_{\mathrm{c}}$ and the hightemperature rutile crystal structure for its $\mathrm{ON}$ state above $T_{\mathrm{c}}$.

To simulate the transport across the $\mathrm{VO}_{2}$ tunnel junction, we construct a $\mathrm{Pd} / \mathrm{HfO}_{2} / \mathrm{VO}_{2}$ supercell. To construct the supercell with different materials (i.e., $\mathrm{VO}_{2} /$ dielectric/metal), we use a slab of $\mathrm{VO}_{2}$ along the [001] orientation in both the rutile (metallic) and $\mathrm{Ml}$ (insulating) phase of $\mathrm{VO}_{2}$. This orientation is the same as that of $\mathrm{VO}_{2}$ used in the experimental tunnel junction structures. Further, this particular choice of orientation removes any dangling bonds at the $\mathrm{VO}_{2}$ interface and eliminates the effect of any resulting surface states. To minimize the number of atoms in the supercell, we use the monoclinic phase of the $\mathrm{HfO}_{2}$ dielectric. The lattice parameters for $\mathrm{HfO}_{2}$ used in the simulation are reported by Zhou et al. ${ }^{17}$ While constructing the supercell, the $b$ and $c$ axes of the $\mathrm{HfO}_{2}$ lattice are swapped, enabling the strain to be minimized. The supercell is then completed by adding [001] Pd. The resulting transverse lattice constants for the supercell are $a=1.404 \mathrm{~nm}, b$ $=1.404 \mathrm{~nm}$ (rutile $\mathrm{VO}_{2}$ supercell) and $a=1.404 \mathrm{~nm}, b=$ $1.7906 \mathrm{~nm}$ ( $\mathrm{M} 1 \mathrm{VO}_{2}$ supercell). In the rutile $\mathrm{VO}_{2}$ phase, the corresponding strain on $\mathrm{HfO}_{2}$ at the $\mathrm{HfO}_{2} /[001]$ rutile $\mathrm{VO}_{2}$ interface is $1.13 \%$, and the strain on $\mathrm{Pd}$ metal at the $\mathrm{Pd} / \mathrm{HfO}_{2}$ interface is $1.79 \%$. The corresponding values of strain for the M1 case are $1.53 \%$ and $1.74 \%$.

As depicted in Figure 6a, we have also considered the transverse directions ( $x y$-plane) as periodic to unite the bulk nature of different materials. To relax the structural interface of this tunnel junction, structure optimization has been done by quasi-Newton scheme ${ }^{18}$ until all the forces acting on atoms become smaller than $0.001 \mathrm{eV} / \AA ̊$. In the transport computations we employ DFT+NEGF to the atomic structure of both the device and the electrodes in a self-consistent manner. The conductance of the device has been calculated as

$$
G=\frac{2 e^{2}}{h} \sum_{k_{\|}} T\left(E, k_{\|}\right)
$$


where $e$ is the electron charge, $h$ is Planck's constant, $k_{\|}$is the Bloch wave vector, and $E$ is the energy. The transmission probabilities $T$ are calculated by

$$
T\left(E, k_{\|}\right)=\operatorname{Tr}\left[\Gamma_{\mathrm{L}} G^{\mathrm{r}} \Gamma_{\mathrm{R}} G^{\mathrm{a}}\right]
$$

in which $G^{\mathrm{r}}$ and $G^{\mathrm{a}}$ are the retarded and advanced Green's functions and $\Gamma_{\mathrm{L}}$ and $\Gamma_{\mathrm{R}}$ are the contact broadening functions associated with the left and right electrodes, respectively, which are anti-Hermitian components of the self-energy terms of the contacts, $\sum_{\mathrm{L}}$ and $\sum_{\mathrm{L}}$, and their conjugates $\sum_{\mathrm{L}}^{\dagger}$ and $\sum_{\mathrm{R}}^{\dagger}\left[\Gamma_{\mathrm{L}, \mathrm{R}}=\right.$ $\left.i\left(\sum_{\mathrm{L}, \mathrm{R}}-\sum_{\mathrm{L}, \mathrm{R}}^{\dagger}\right) / 2\right] .{ }^{19}$ As our simulated device setup has a perfect periodicity in the plane perpendicular ( $x y$-plane) to the transport direction ( $z$-direction), the Bloch wave vector along $k_{\|}=\left(k_{x}, k_{y}\right)$ is preserved, and the total tunneling conductance is defined by the integral over the $2 \mathrm{D}$ Brillouin zone in the $k_{\|}$ plane.

Figure $6 \mathrm{~b}$ shows the schematic of the tunneling mechanism in $\mathrm{VO}_{2}$ based tunnel junction when $\mathrm{VO}_{2}$ is in its insulating $\mathrm{M} 1$ state. As $\mathrm{VO}_{2}$ opens a bandgap when lowering the temperature, eliminating the electronic states in the $\mathrm{VO}_{2}$ around the Fermi energy, the external voltage has to be high enough to compensate for the missing electronic states near the Fermi energy in $\mathrm{VO}_{2}$ to allow transport through the structure. This is also reflected in the transmission (Figure $6 \mathrm{c}$ ).

Above $T_{\mathcal{o}}$, when $\mathrm{VO}_{2}$ is in its metallic rutile crystal structure, electronic states throughout the whole structure line up (Figure $6 \mathrm{~d}$ ), allowing for controlled direct tunneling from the Pd-layer through the tunnel barrier into the metallic $\mathrm{VO}_{2}$ layer (Figure 6e). When comparing the experimentally measured $G$ vs $V$ traces (Figure 7a) in both ON and OFF states, we find that the signature shape of the computed quantum mechanical tunnel conductance (Figure $7 \mathrm{~b}$ ) agrees well with our measurement results.
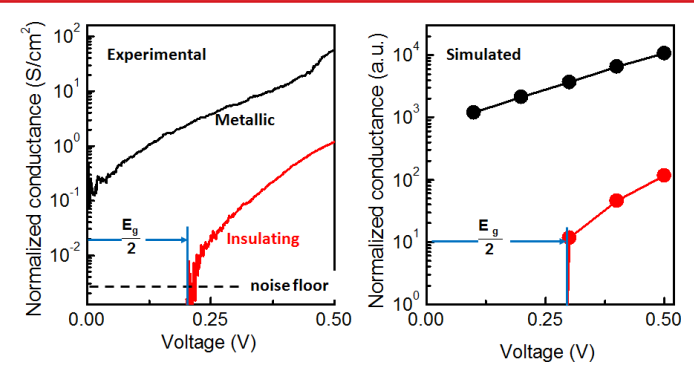

Figure 7. (a) Experimental and (b) simulated area normalized conductance vs $V$ traces for both the metallic and the insulating phase of $\mathrm{VO}_{2}$.

In conclusion, we are able to accurately measure $I V$ traces through distinct, individual $\mathrm{VO}_{2}$ nanopillars while also allowing the benefit of topographic imaging. In the future this might allow us to simultaneously investigate the topographic phase transition along with the electronic phase transition in $\mathrm{VO}_{2}$ that we investigate in this work. The $\mathrm{VO}_{2}$ nanotunnel junctions show an abrupt change in conductance of approximately 2 orders of magnitude when $\mathrm{VO}_{2}$ undergoes its phase transition from the insulating (OFF) state to its metallic (ON) state. This MIT occurs at $T_{c}=287.5 \pm 7 \mathrm{~K}$. When $\mathrm{VO}_{2}$ is in the electrically insulating state, the $I V$ traces show a region of suppressed current (Hubbard gap) of a width of $0.4 \pm 0.07 \mathrm{~V}$. We have performed ab initio transport calculations through DFT+NEGF formalism by considering both rutile and M1 phase for the ON and OFF states, respectively. To incorporate the Mott properties in the M1 phase, we use $U=4 \mathrm{eV}$ which results in a very good agreement between the experimental Hubbard gap value and calculated bandgap value of $0.56 \mathrm{eV}$. Our theoretical quantum mechanical transport calculations show very good agreement with the experimental results in terms of the shape of the $G V$ traces. Our results are a step toward the future use of correlated oxide based tunnel junctions with high on-off conductance ratios for both memory and logic applications.

\section{AUTHOR INFORMATION}

\section{Corresponding Author}

*E-mail: huefner@phys.ethz.ch.

\section{Present Address}

M.H.: Department of Applied Physics, University of Tuebingen, 72076 Tuebingen, Germany.

\section{Notes}

The authors declare no competing financial interest.

\section{ACKNOWLEDGMENTS}

M.H. thanks the DFG fellowship (HU-1960/1-1) for their support. All authors would like to thank Prof. Hoffman (Harvard University) for allowing the use of her facilities to carry out the measurements. R.G. and S.D. thank the Center for Low Energy Systems Technology (LEAST), one of the six SRC STARnet Centers, sponsored by MARCO and DARPA for their financial support. NS, HP, and DGS were funded by Office of Naval Research through award N00014-11-1-0665. This work was performed in part at the Cornell NanoScale Facility, a member of the National Nanotechnology Infrastructure Network, which is supported by the National Science Foundation (Grant No. ECCS-0335765).

\section{REFERENCES}

(1) Rosevear, W. H.; Paul, W. Phys. Rev. B 1973, 7, 2109.

(2) Morin, F. J. Phys. Rev. Lett. 1959, 3, 34.

(3) Zimmers, A.; Aigouy, L.; Mortier, M.; Sharoni, A.; Wang, S.; West, K. G.; Ramirez, J. G.; Schuller, I. K. Phys. Rev. Lett. 2013, 110, 056601 .

(4) Cavalleri, A.; Dekorsy, T.; Chong, H. W.; Kieffer, J.-C.; Schoenlein, R. W. Phys. Rev. B 2004, 70, 161102.

(5) Cao, J.; Ertekin, E.; Srinivasan, V.; Fan, W.; Huang, S.; Zheng, H.; Yim, J. W. L.; Khanal, D. R.; Ogletree, D. F.; Grossman, J. C. Nat. Nanotechnol. 2009, 4, 732.

(6) Kübler, C.; Ehrke, H.; Huber, R; Lopez, R.; Halabica, A.; Haglund, R. F., Jr.; Leitenstorfer, A. Phys. Rev. B 2007, 99, 116401.

(7) Verleur, H. W.; Barker, A. S., Jr.; Berglund, C. N. Phys. Rev. 1968, 172, 788 .

(8) Freeman, E.; Kar, A.; Shukla, N.; Misra, R.; Engel-Herbert, R.; Schlom, D.; Gopalan, V.; Rabe, K.; Datta, S. Device Research Conference (DRC), 70th Annual, 2012; pp 243-244.

(9) Sze, S. M.; Ng, K. K. Physics of Semiconductor Devices, 3rd ed. Wiley: Hoboken, NJ, 2007.

(10) Martens, K.; Radu, I. P.; Mertens, S.; Shi, X.; Nyns, L.; Cosemans, S.; Favia, P.; Bender, H.; Conard, T.; Schaekers, M. J. Appl. Phys. 2012, 112, 124501.

(11) Yin, W.; West, K. G.; Lu, J. W.; Pei, Y.; Wolf, S. A.; Reinke, P.; Sun, Y. J. Appl. Phys. 2009, 105, 114322.

(12) QuantumWise simulator, Atomistix ToolKit (ATK); http:// www.quantumwise.com/ (accessed September 2014).

(13) Eyert, V. Ann. Phys. (Leipzig) 2002, 11.

(14) Quackenbush, N. F.; Tashman, J. W.; Mundy, J. A.; Sallis, S.; Paik, H.; Misra, R.; Moyer, J. A.; Guo, J.-H.; Fischer, D. A.; Woicik, J. 
C.; Muller, D. A.; Schlom, D. G.; Piper, L. F. J. Nano Lett. 2013, 13, 4857.

(15) Kim, S.; Kim, K.; Kang, C.-J.; Min, B. I. Phys. Rev. B 2013, 87, 195106.

(16) Huffman, T. J.; Xu, P.; Qazilbash, M. M.; Walter, E. J.; Krakauer, H.; Wei, J.; Cobden, D. H.; Bechtel, H. A.; Martin, M. C.; Carr, G. L.; Basov, D. N. Phys. Rev. B 2013, 87, 115121.

(17) Zhou, B.; Shi, H.; Zhang, X. D.; Su, Q.; Jiang, Z. Y. J. Phys. D: Appl. Phys. 2014, 47, 115502.

(18) Sheppard, D.; Terrell, R.; Henkelman, G. J. Chem. Phys. 2008, $128,134106$.

(19) Datta, S.; Quantum Transport-Atom to Transistor; Cambridge University Press: Cambridge, 2005. 